This item was submitted to Loughborough's Research Repository by the author.

Items in Figshare are protected by copyright, with all rights reserved, unless otherwise indicated.

\title{
Solving DEA models in a single optimization stage: Can the non- Archimedean infinitesimal be replaced by a small finite epsilon?
}

PLEASE CITE THE PUBLISHED VERSION

http://dx.doi.org/10.1016/j.ejor.2016.09.022

PUBLISHER

(C) Elsevier

VERSION

AM (Accepted Manuscript)

\section{PUBLISHER STATEMENT}

This work is made available according to the conditions of the Creative Commons Attribution-NonCommercialNoDerivatives 4.0 International (CC BY-NC-ND 4.0) licence. Full details of this licence are available at: https://creativecommons.org/licenses/by-nc-nd/4.0/

\section{LICENCE}

CC BY-NC-ND 4.0

\section{REPOSITORY RECORD}

Podinovski, Victor, and Tatiana Bouzdine-Chameeva. 2016. "Solving DEA Models in a Single Optimization Stage: Can the Non-archimedean Infinitesimal Be Replaced by a Small Finite Epsilon?”. figshare. https://hdl.handle.net/2134/22659. 


\title{
Solving DEA models in a single optimization stage: Can the non-Archimedean infinitesimal be replaced by a small finite epsilon?
}

\author{
Victor V. Podinovskia,*, Tatiana Bouzdine-Chameeva ${ }^{\mathrm{b}}$ \\ ${ }^{a}$ School of Business and Economics, Loughborough University, Loughborough LE11 3TU, UK \\ ${ }^{b}$ KEDGE Business School, 680 cours de la Libération, 33405 Talence Cedex, France
}

\begin{abstract}
Single-stage DEA models aim to assess the input or output radial efficiency of a decision making unit and potential mix inefficiency in a single optimization stage. This is achieved by incorporating the sum of input and output slacks, multiplied by a small (theoretically nonArchimedean infinitesimal) value epsilon in the envelopment model or, equivalently, by using this value as the lower bound on the input and output weights in the dual multiplier model. When this approach is used, it is common practice to select a very small value for epsilon. This is based on the expectation that, for a sufficiently small epsilon, the radial efficiency and optimal slacks obtained by solving the single-stage model should be approximately equal to their true values obtained by the two separate optimization stages. However, as well-known, selecting a small epsilon may lead to significant computational inaccuracies. In this paper we prove that there exists a threshold value, referred to as the effective bound, such that, if epsilon is smaller than this bound, the solution to the single-stage program is not approximate but precise (exactly the same as in the two-stage approach), provided there are no computational errors.
\end{abstract}

Keywords: data envelopment analysis, single-stage optimization, effective bound

\section{Introduction}

The two conventional models of data envelopment analysis (DEA) are stated under the assumptions of constant (CRS) and variable (VRS) returns to scale (Charnes et al. 1978, Banker et al. 1984). Both models aim at assessing the input or output radial efficiency of decision making units (DMUs). If a DMU is radial efficient, it may still allow some nonradial, i.e., individual, improvements to its inputs or outputs, in which case it is said to exhibit mix inefficiency (Cooper et al. 2007). A DMU is strongly efficient if and only if its input or output radial efficiency is equal to 1 and it does not exhibit mix inefficiency.

The issues of input and output slacks and ways to account for them in efficiency assessment were raised and discussed in the early DEA literature (Charnes et al. 1979, Boyd and Färe 1984, Charnes and Cooper 1984). Following Ali and Seiford (1993a), the testing of radial and mix efficiency of any $\mathrm{DMU}_{o}$ is usually performed in two stages. The first stage evaluates the input or output radial efficiency of $\mathrm{DMU}_{o}$ and identifies its radial projection on the boundary of the technology. The second stage tests for mix efficiency by maximizing the sum of component slacks associated with individual inputs and outputs.

\footnotetext{
*Corresponding author

Email addresses: v.podinovski@lboro.ac.uk (Victor V. Podinovski), tatiana.chameeva@kedgebs.com (Tatiana Bouzdine-Chameeva)
} 
An alternative to the two-stage solution is the single-stage approach that accounts for both radial and individual component improvements to $\mathrm{DMU}_{o}$ in a single program. According to this approach, the objective function of the envelopment CRS or VRS model, representing an input or output radial improvement factor, is modified to include the sum of input and output slacks multiplied by a very small (theoretically infinitesimal) constant $\epsilon>0$. Equivalently, in the dual multiplier model a lower bound $\epsilon$ is incorporated on all input and output weights.

As shown by Ali and Seiford (1993b), the single-stage solution approach has important theoretical and computational drawbacks. From a theoretical perspective, the constant $\epsilon$ has to be sufficiently small for the multiplier model to be feasible or, equivalently, for the envelopment model to have a finite optimum value. Furthermore, even if $\epsilon$ is small and the resulting models have a finite optimal solution, there is no guarantee that the estimated radial efficiency and component slacks are close to their true values obtained using the twostage approach. Intuitively, the latter concern can be addressed (and the precision of the approximate solution improved) by taking $\epsilon$ as a very small value. However, specifying a very small $\epsilon$ leads to well-documented computational problems arising from finite numerical tolerance of optimization software (Ali and Seiford 1993b). ${ }^{1}$

Despite the above drawbacks, solving DEA models in a single stage has attracted significant attention in the literature. The most common question addressed in this strand of research concerns estimation of the assurance interval $\left[0, \epsilon^{*}\right]$, where $\epsilon^{*}>0$ is the maximum value $\epsilon$ that keeps the multiplier models feasible (Ali and Seiford 1993b, Mehrabian et al. 2000, Amin and Toloo 2004, Alirezaee 2005, MirHassani and Alirezaee 2005). Clearly, choosing an $\epsilon$ from the assurance region does not guarantee that the resulting single-stage optimal solution is a good approximation of the true radial efficiency of $\mathrm{DMU}_{o}$ and the corresponding component slacks.

The contribution of our paper is different from the above literature. We prove that, for each single-stage model, there exists a strictly positive effective bound $\epsilon_{o} \leq \epsilon^{*}$ such that, theoretically, the single-stage model with any $\epsilon \in\left(0, \epsilon_{o}\right)$ yields the true radial efficiency and component slacks for $\mathrm{DMU}_{o}$. The latter are exactly the same as assessed by the two separate optimization stages. We refer to the range $\left(0, \epsilon_{o}\right)$ as the effective interval. For all $\epsilon \in\left(0, \epsilon_{o}\right)$, the single-stage approach is, at least theoretically, not an approximate, but a precise solution method for simultaneous assessment of the radial and mix efficiency of DMUs.

These theoretical results show that, for all sufficiently small values $\epsilon$, any difference between the true efficiency of $\mathrm{DMU}_{o}$ and its estimate obtained from a single-stage model are entirely due to computational, and not approximation, errors. As an illustration, we consider numerical examples in which computational inaccuracies are negligible (i.e., the preciseness of the optimal solution is confirmed by observation of a graph). In these examples, strictly in line with the theoretical results, the single-stage programs with $\epsilon<\epsilon_{o}$ produce efficiency scores and optimal slacks identical to the two-stage optimization approach.

Using known results of sensitivity analysis in linear optimization (Roos et al. 2005), we show that the effective bound $\epsilon_{o}$ can be evaluated as the optimum value of a specially constructed linear program. Based on this, we prove that $\epsilon_{o}$ depends on $\mathrm{DMU}_{o}$ and the assumption of returns to scale (CRS or VRS), and is generally different for models in the input and output orientation. We also prove that the effective bound $\epsilon_{o}$ in a DEA model under the assumption of CRS does not exceed the effective bound in the similarly specified

\footnotetext{
${ }^{1}$ Improved computational algorithms for solving DEA models with an infinitesimal bound on multiplier weights have been developed by Charnes et al. (1992, 1993). Scheel and Scholtes (2003, page 154) show that numerical problems can also occur with the two-stage optimization approach.
} 
model under the assumption of VRS.

\section{Preliminaries}

Let $\mathcal{T}_{\mathrm{CRS}}$ and $\mathcal{T}_{\text {VRS }}$ be the CRS and VRS technologies generated by a finite set of observed DMUs $\left(X_{j}, Y_{j}\right), j=1, \ldots, n$. In this notation, $X_{j} \in \mathbb{R}_{+}^{m}$ is the vector of inputs and $Y_{j} \in \mathbb{R}_{+}^{s}$ is the vector of outputs. ${ }^{2}$ Let $\bar{X}$ and $\bar{Y}$ denote the $m \times n$ and $s \times n$ matrices whose columns are the vectors $X_{j}$ and $Y_{j}, j=1, \ldots, n$.

$\operatorname{DMU}(X, Y)$ in technology $\mathcal{T}_{\mathrm{CRS}}$ or $\mathcal{T}_{\text {VRS }}$ is strongly efficient if there does not exist a $\operatorname{DMU}\left(X^{\prime}, Y^{\prime}\right)$ in the same technology, such that $X^{\prime} \leq X, Y^{\prime} \geq Y$, and $\left(X^{\prime}, Y^{\prime}\right) \neq(X, Y)$.

Let $\left(X_{o}, Y_{o}\right)$ be the DMU in either technology whose efficiency is being evaluated. This DMU may be observed or unobserved, ${ }^{3}$ but we require that $X_{o} \neq \mathbf{0}$ and $Y_{o} \neq \mathbf{0}$. (Throughout this paper we use bold notation $\mathbf{0}$ and $\mathbf{1}$ to denote vectors of zeroes and ones of appropriate dimensions.)

\section{Effective bounds for the output-oriented CRS model}

Below we consider the case of output-oriented CRS model in detail. The input-oriented CRS models and the case of VRS require a straightforward adjustment to this development and are briefly outlined in the subsequent sections.

\subsection{The two-stage solution approach}

The output radial efficiency of $\operatorname{DMU}\left(X_{o}, Y_{o}\right)$ is the inverse of the optimal value $\eta^{*}$ in the following output-oriented envelopment CRS model:

$$
\begin{aligned}
\eta^{*}=\max & \eta \\
\text { subject to } & \bar{X} \lambda+S_{X}=X_{o} \\
& -\bar{Y} \lambda+S_{Y}=-\eta Y_{o}, \\
& \lambda, S_{X}, S_{Y} \geq \mathbf{0}, \eta \text { sign free, }
\end{aligned}
$$

where $S_{X} \in \mathbb{R}_{+}^{m}$ and $S_{Y} \in \mathbb{R}_{+}^{s}$ are vectors of input and output slacks, respectively.

After program (1) is solved and the maximum output improvement factor $\eta^{*}$ is found, the second-stage program is solved:

$$
\begin{array}{cl}
\sigma^{*}=\max & \mathbf{1}^{\top} S_{X}+\mathbf{1}^{\top} S_{Y} \\
\text { subject to } & \bar{X} \lambda+S_{X}=X_{o}, \\
& -\bar{Y} \lambda+S_{Y}=-\eta^{*} Y_{o} \\
& \lambda, S_{X}, S_{Y} \geq \mathbf{0} .
\end{array}
$$

It is known (see, e.g., Cooper et al. 2007) that DMU $\left(X_{o}, Y_{o}\right)$ is strongly efficient if and only if $\eta^{*}=1$ and $\sigma^{*}=0$. Define

$$
\left(X^{*}, Y^{*}\right)=\left(\bar{X} \lambda^{*}, \bar{Y} \lambda^{*}\right)=\left(X_{o}-S_{X}^{*}, \eta^{*} Y_{o}+S_{Y}^{*}\right)
$$

$\operatorname{DMU}\left(X^{*}, Y^{*}\right)$ is referred to as an efficient target for DMU $\left(X_{o}, Y_{o}\right)$. It is straightforward to verify that $\mathrm{DMU}\left(X^{*}, Y^{*}\right)$ is strongly efficient in technology $\mathcal{T}_{\mathrm{CRS}}$.

Remark 1. Let $\left\langle\lambda^{*}, S_{X}^{*}, S_{Y}^{*}\right\rangle$ be any optimal solution to (2). Then $\left\langle\eta^{*}, \lambda^{*}, S_{X}^{*}, S_{Y}^{*}\right\rangle$ is an optimal solution to program (1).

\footnotetext{
${ }^{2}$ We assume that each observed input vector $X_{j}, j=1, \ldots, n$, has at least one strictly positive component. This assumption is used in the proof of Lemma 1.

${ }^{3}$ An unobserved DMU is an element of technology $\mathcal{T}_{\text {CRS }}$ or $\mathcal{T}_{\text {VRS }}$ which is not one of the observed DMUs. For example, in both technologies the weighted averages (convex combinations) of observed DMUs are typically unobserved DMUs.
} 


\subsection{The single-stage solution approach}

The single-stage approach combines the maximization of the output-improvement factor $\eta$ as the primary objective, and the sum of input and output slacks as the secondary objective, in a single linear program.

Consider some (typically very small) $\epsilon>0$. The single-stage envelopment program is stated as follows:

$$
\begin{aligned}
\max & \eta+\epsilon\left(\mathbf{1}^{\top} S_{X}+\mathbf{1}^{\top} S_{Y}\right) \\
\text { subject to } & \bar{X} \lambda+S_{X}=X_{o} \\
& -\bar{Y} \lambda+S_{Y}=-\eta Y_{o}, \\
& \lambda, S_{X}, S_{Y} \geq \mathbf{0}, \eta \text { sign free. }
\end{aligned}
$$

Its dual multiplier program assumes the form

$$
\begin{aligned}
\min & X_{o}^{\top} v \\
\text { subject to } & Y_{o}^{\top} u=1, \\
& \bar{X}^{\top} v-\bar{Y}^{\top} u \geq \mathbf{0}, \\
& u, v \geq \epsilon \mathbf{1} .
\end{aligned}
$$

It is known that, if $\epsilon$ is relatively large, program (4) is infeasible and, by duality, program (3) has an unbounded optimum value. ${ }^{4}$ Mehrabian et al. (2000) develop a linear programming approach that identifies the maximum value $\epsilon^{*}$ for which program (4), and therefore (3), have a finite optimal solution. The range $\left[0, \epsilon^{*}\right]$ is referred to as the assurance interval, which is specific to $\operatorname{DMU}\left(X_{o}, Y_{o}\right)$. Any value $\epsilon \in\left[0, \epsilon^{*}\right]$ is referred to as an assurance value.

Assuming $\epsilon \in\left(0, \epsilon^{*}\right]$, let

$$
\left\langle\eta^{\epsilon}, \lambda^{\epsilon}, S_{X}^{\epsilon}, S_{Y}^{\epsilon}\right\rangle
$$

be any optimal solution to program (3) for the given $\epsilon .^{5}$ Because (5) is a feasible solution of program (1), we always have

$$
\eta^{\epsilon} \leq \eta^{*}
$$

Definition 1. We call $\epsilon>0$ an effective value for DMU $\left(X_{o}, Y_{o}\right)$ if

(i) program (3) and, therefore, its dual (4) have a finite optimal solution;

(ii) for any optimal solution (5) of program (3), we have $\eta^{\epsilon}=\eta^{*}$, where $\eta^{*}$ is the optimum value of program (1).

The significance of the above definition is underlined by the next result.

\footnotetext{
${ }^{4}$ The economic meaning of infeasibility of program (4) follows from the work of Podinovski and BouzdineChameeva $(2013,2015)$. The constraints $u, v \geq \epsilon \mathbf{1}$ of program (8) are absolute weight restrictions. After a rearrangement (Podinovski 2004, 2005), these are interpretable as production trade-offs that expand the underlying technology $\mathcal{T}_{\mathrm{CRS}}$. The results of Podinovski and Bouzdine-Chameeva (2013) imply that, if program (8) is infeasible, the constraints $u, v \geq \epsilon \mathbf{1}$ generate free production of output vectors in the expanded technology. The meaning of optimal input and output weights in DEA models with weight restrictions has recently been explored by Podinovski (2016).

${ }^{5}$ Program (3) may have multiple optimal solutions (5), and optimal $\eta^{\epsilon}$ may not be unique. This is highlighted by Proposition 4 and illustrated by Example 1 .
} 
Proposition 1. Let $\epsilon$ be an effective value for $D M U\left(X_{o}, Y_{o}\right)$, and let (5) be any optimal solution to program (3). Then (5) is an optimal solution to program (1), and $\left\langle\lambda^{\epsilon}, S_{X}^{\epsilon}, S_{Y}^{\epsilon}\right\rangle$ is an optimal solution to program (2). Conversely, let $\eta^{*}$ be the optimum value of program (1), and let $\left\langle\lambda^{*}, S_{X}^{*}, S_{Y}^{*}\right\rangle$ be any optimal solution to (2). Then $\left\langle\eta^{*}, \lambda^{*}, S_{X}^{*}, S_{Y}^{*}\right\rangle$ is an optimal solution to $(3)$.

The proofs of Proposition 1 and the other statements are given in Appendix A.

Reinterpreting the above result, if $\epsilon$ is an effective value for DMU $\left(X_{o}, Y_{o}\right)$, then assessing the efficiency of DMU $\left(X_{o}, Y_{o}\right)$ by solving the single-stage program (3) is theoretically equivalent to assessing its efficiency in two stages, by solving two consecutive programs (1) and (2).

Below we prove that the set of all effective values $\epsilon$ for DMU $\left(X_{o}, Y_{o}\right)$ is the open interval $\left(0, \epsilon_{o}\right)$, where $\epsilon_{o}>0$ can be computed as the optimum value of a specially constructed linear program. We refer to $\left(0, \epsilon_{o}\right)$ as the effective interval, and call $\epsilon_{o}$ the effective bound for DMU $\left(X_{o}, Y_{o}\right)$. Note that $\epsilon_{o}$ is excluded from the effective interval and, as proved below, is not itself an effective value.

\subsection{Calculating the effective bound $\epsilon_{o}$}

Consider the following linear program, where $\eta^{*}$ and $\sigma^{*}$ are the optimum values of programs (1) and (2), respectively:

$$
\begin{aligned}
\varphi_{o}=\min & Y_{o}^{\top} u \\
\text { subject to } & X_{o}^{\top} v-\eta^{*} Y_{o}^{\top} u=\sigma^{*}, \\
& \bar{X}^{\top} v-\bar{Y}^{\top} u \geq \mathbf{0}, \\
& u, v \geq \mathbf{1}
\end{aligned}
$$

Proposition 2. Program (7) is feasible and has a finite optimum value $\varphi_{o} \geq Y_{o}^{\top} \mathbf{1}$.

Theorem 1. An $\epsilon>0$ is an effective value for $D M U\left(X_{o}, Y_{o}\right)$ if and only if $\epsilon<1 / \varphi_{o}$. Therefore, $\epsilon_{o}=1 / \varphi_{o}>0$ is the effective bound, and $\left(0, \epsilon_{o}\right)$ is the effective interval for DMU $\left(X_{o}, Y_{o}\right)$.

As follows from Theorem 1 , any $\epsilon \geq 1 / \varphi_{o}$ is not an effective value for $\operatorname{DMU}\left(X_{o}, Y_{o}\right)$. The following two results provide a further clarification.

Proposition 3. Consider any $\epsilon>1 / \varphi_{o}$ such that program (3) has a finite optimum value, i.e., $\epsilon \in\left(1 / \varphi_{o}, \epsilon^{*}\right]$. Then, for any optimal solution (5) of program (3), $\eta^{\epsilon}<\eta^{*}$.

Proposition 4. Let $\epsilon=1 / \varphi_{o}$. Then $\left\langle\eta^{*}, \lambda^{*}, S_{X}^{*}, S_{Y}^{*}\right\rangle$ is an optimal solution to program (3). Furthermore, there exists an $\bar{\alpha} \sim_{\tilde{S}_{X}} 0$ such that, for any $\alpha \in(0, \bar{\alpha}]$, program (3) has an alternative optimal solution $\left\langle\tilde{\eta}, \tilde{\lambda}, \tilde{S}_{X}, \tilde{S}_{Y}\right\rangle$ to program (3) such that $\tilde{\eta}=\eta^{*}-\alpha$.

The above results are illustrated by numerical examples in Section 6 .

Remark 2. If DMU $\left(X_{o}, Y_{o}\right)$ is strongly efficient, we have $\eta^{*}=1$ and $\sigma^{*}=0$. Then program (7) assumes the form

$$
\begin{aligned}
\varphi_{o}=\min & Y_{o}^{\top} u \\
\text { subject to } & X_{o}^{\top} v-Y_{o}^{\top} u=0 \\
& \bar{X}^{\top} v-\bar{Y}^{\top} u \geq \mathbf{0} \\
& u, v \geq \mathbf{1} \\
& 5
\end{aligned}
$$


Taking into account the first of the above constraints, we can change the objective function of program (8) to $X_{o}^{\top} v$. Similarly, we can change the objective function of program (7) to $\left(X_{o}^{\top} v-\sigma^{*}\right) / \eta^{*}$.

Remark 3. Let $\epsilon_{o}$ and $\hat{\epsilon}_{o}$ be, respectively, the effective bounds for DMU $\left(X_{o}, Y_{o}\right)$ and its output radial target $(\hat{X}, \hat{Y})=\left(X_{o}, \eta^{*} Y_{o}\right)$. ${ }^{6}$ We have the following result:

Proposition 5. $\hat{\epsilon}_{o}=\epsilon_{o} / \eta^{*}$.

Example 1 in Section 6 illustrates Proposition 5.

\section{Effective bounds for the input-oriented CRS model}

An extension of the above development to the case of input orientation is straightforward. In order to avoid repetition, we briefly outline only the main results.

The input radial efficiency of $\operatorname{DMU}\left(X_{o}, Y_{o}\right)$ is found as the optimal value $\theta^{*}$ in the following program:

$$
\begin{aligned}
\theta^{*}=\min & \theta \\
\text { subject to } & -\bar{X} \lambda-S_{X}=-\theta X_{o}, \\
& \bar{Y} \lambda-S_{Y}=Y_{o}, \\
& \lambda, S_{X}, S_{Y} \geq \mathbf{0}, \theta \text { sign free. }
\end{aligned}
$$

The second-stage program takes the following form, where $\theta^{*}$ is the optimum value of $(9)$ :

$$
\begin{array}{cl}
\tau^{*}=\max & \mathbf{1}^{\top} S_{X}+\mathbf{1}^{\top} S_{Y} \\
\text { subject to } & -\bar{X} \lambda-S_{X}=-\theta^{*} X_{o} \\
& \bar{Y} \lambda-S_{Y}=Y_{o} \\
& \lambda, S_{X}, S_{Y} \geq \mathbf{0} .
\end{array}
$$

The single-stage input-oriented program is stated as follows: ${ }^{7}$

$$
\begin{aligned}
\min & \theta-\epsilon\left(\mathbf{1}^{\top} S_{X}+\mathbf{1}^{\top} S_{Y}\right) \\
\text { subject to } & -\bar{X} \lambda-S_{X}=-\theta X_{o}, \\
& \bar{Y} \lambda-S_{Y}=Y_{o}, \\
& \lambda, S_{X}, S_{Y} \geq \mathbf{0}, \theta \text { sign free. }
\end{aligned}
$$

Its dual multiplier program is

$$
\begin{aligned}
\max & Y_{o}^{\top} u \\
\text { subject to } & X_{o}^{\top} v=1, \\
& -\bar{X}^{\top} v+\bar{Y}^{\top} u \leq \mathbf{0}, \\
& u, v \geq \epsilon \mathbf{1}
\end{aligned}
$$

Definition 2. In the case of input minimization, $\epsilon>0$ is an effective value for DMU $\left(X_{o}, Y_{o}\right)$ if

\footnotetext{
${ }^{6}$ Note that the output radial target $(\hat{X}, \hat{Y})$ is strongly efficient if and only if DMU $\left(X_{o}, Y_{o}\right)$ does not exhibit mix inefficiency, i.e., the optimal value $\sigma^{*}$ of program (2) is equal to zero.

${ }^{7}$ Note that the term with sum of slacks in the objective function of (11) is stated with the negative sign.
} 
(i) program (11) and, therefore, its dual (12) have a finite optimal solution;

(ii) for any optimal solution $\left\langle\theta^{\epsilon}, \lambda^{\epsilon}, S_{X}^{\epsilon}, S_{Y}^{\epsilon}\right\rangle$ of program (11), we have $\theta^{\epsilon}=\theta^{*}$, where $\theta^{*}$ is the optimum value of program (9).

Restating and proving an input-oriented analogue of Proposition 1 is straightforward, and is not given. In particular, if $\epsilon$ is an effective value, solving the single-stage inputoriented program (11) is equivalent to assessing the input radial efficiency of DMU $\left(X_{o}, Y_{o}\right)$ and the corresponding optimal slacks in two stages (9) and (10).

Consider the following linear program, where $\theta^{*}$ and $\tau^{*}$ are the optimum values of programs (9) and (10), respectively:

$$
\begin{aligned}
\tilde{\varphi}_{o}=\min & X_{o}^{\top} v \\
\text { subject to } & \theta^{*} X_{o}^{\top} v-Y_{o}^{\top} u=\tau^{*}, \\
& \bar{X}^{\top} v-\bar{Y}^{\top} u \geq \mathbf{0}, \\
& u, v \geq \mathbf{1} .
\end{aligned}
$$

Theorem 2. For the single-stage input-oriented program (11), $\epsilon>0$ is an effective value if and only if $\epsilon<1 / \tilde{\varphi}_{o}$. Therefore, $\tilde{\epsilon}_{o}=1 / \tilde{\varphi}_{o}>0$ is the effective bound, and $\left(0, \tilde{\epsilon}_{o}\right)$ is the effective interval for $\operatorname{DMU}\left(X_{o}, Y_{o}\right)$.

The proof of the Theorem 2 follows closely the proof of Theorem 1 and is not given. ${ }^{8}$ We also have direct analogues of Proposition 3 and 4. In particular, the latter implies that, if $\epsilon=1 / \tilde{\varphi}_{o}$, program (11) has multiple optimal solutions $\left\langle\theta^{\epsilon}, \lambda^{\epsilon}, S_{X}^{\epsilon}, S_{Y}^{\epsilon}\right\rangle$, and there exist optimal solutions with $\theta^{\epsilon}=\theta^{*}$ and $\theta^{\epsilon}>\theta^{*}$.

Furthermore, for the input radial projection $(\hat{X}, \hat{Y})=\left(\theta^{*} X_{o}, Y_{o}\right)$ of DMU $\left(X_{o}, Y_{o}\right)$, an analogue of Proposition 5 is true. Namely, we have $\hat{\epsilon}_{o}=\tilde{\epsilon}_{o} / \theta^{*}$, where $\tilde{\epsilon}_{o}$ and $\hat{\epsilon}_{o}$ are the effective bounds for DMUs $\left(X_{o}, Y_{o}\right)$ and $(\hat{X}, \hat{Y})$, respectively.

Remark 4. Generally, the effective bound $\epsilon_{o}$ is different for the output-oriented and inputoriented programs (3) and (11). An exception to this is highlighted by the following result.

Proposition 6. Let DMU $\left(X_{o}, Y_{o}\right)$ be strongly efficient in technology $\mathcal{T}_{\mathrm{CRS}}$. Then $\varphi_{o}=\tilde{\varphi}_{o}$, which implies $\epsilon_{o}=\tilde{\epsilon}_{o}$.

\section{Effective bounds for the VRS models}

Under the assumption of VRS, the two-stage output-oriented programs and the corresponding single-stage program are obtained from programs (1), (2) and (3), by incorporating the additional normalizing equality

$$
\mathbf{1}^{\top} \lambda=1
$$

The dual to the single-stage VRS program incorporates the additional sign-free variable $\omega$ dual to the normalizing equality (14):

$$
\begin{aligned}
\min & X_{o}^{\top} v+\omega \\
\text { subject to } & Y_{o}^{\top} u=1, \\
& \bar{X}^{\top} v-\bar{Y}^{\top} u+\omega \mathbf{1} \geq \mathbf{0}, \\
& u, v \geq \epsilon \mathbf{1}, \omega \text { sign free. }
\end{aligned}
$$

\footnotetext{
${ }^{8}$ As in the latter case, the proof implies that program (13) is feasible and has a finite optimum value $\tilde{\varphi}_{o} \geq X_{o}^{\top} \mathbf{1}$. The latter means that $\tilde{\epsilon}_{o} \leq 1 / X_{o}^{\top} \mathbf{1}$.
} 
Adapting Definition 1, we refer to $\epsilon$ as an effective value for the single-stage outputoriented VRS program (program (3) with the normalizing condition (14)) if, for any of its optimal solutions $\left\langle\eta^{\epsilon}, \lambda^{\epsilon}, S_{X}^{\epsilon}, S_{Y}^{\epsilon}\right\rangle$, we have $\eta^{\epsilon}=\eta^{*}$.

Consider the following linear program, where $\eta^{*}$ and $\sigma^{*}$ are the optimum values of the VRS analogues of programs (1) and (2) (that incorporate the additional equality (14)):

$$
\begin{aligned}
\varphi_{o}=\min & Y_{o}^{\top} u \\
\text { subject to } & X_{o}^{\top} v-\eta^{*} Y_{o}^{\top} u+\omega=\sigma^{*}, \\
& \bar{X}^{\top} v-\bar{Y}^{\top} u+\omega \mathbf{1} \geq \mathbf{0}, \\
& u, v \geq \mathbf{1}, \omega \operatorname{sign} \text { free. }
\end{aligned}
$$

It is straightforward to show that Theorem 1 and Propositions 1-5 remain true in the case of VRS, with obvious modifications. (In their statements, programs (1) and (3) are replaced by their VRS analogues, and $\varphi_{o}$ is the optimum value of program (15).) In particular, for the single-stage output-oriented VRS program, the effective interval is $\left(0, \epsilon_{o}\right)$, where $\epsilon_{o}=1 / \varphi_{o}$.

Similarly, in the case of input orientation, let $\theta^{*}$ be the input radial efficiency of DMU $\left(X_{o}, Y_{o}\right)$ in the VRS technology, and $\tau^{*}$ be the corresponding maximum sum of input and output slacks. Then the effective interval for the single-stage input-oriented VRS program is $\left(0, \tilde{\epsilon}_{o}\right)$, where $\tilde{\epsilon}_{o}$ is the inverse of the optimum value $\tilde{\varphi}_{o}$ in the following program:

$$
\begin{aligned}
\tilde{\varphi}_{o}=\min & X_{o}^{\top} v \\
\text { subject to } & \theta^{*} X_{o}^{\top} v-Y_{o}^{\top} u+\omega=\tau^{*}, \\
& \bar{X}^{\top} v-\bar{Y}^{\top} u+\omega \mathbf{1} \geq \mathbf{0}, \\
& u, v \geq \mathbf{1}
\end{aligned}
$$

The next result shows that the effective bound in the CRS model does not exceed the effective bound in the VRS model, in both input and output orientations. Let DMU $\left(X_{o}, Y_{o}\right) \in \mathcal{T}_{\text {VRS }}$ and, therefore, $\left(X_{o}, Y_{o}\right) \in \mathcal{T}_{\text {CRS }}$. Denote $\epsilon_{o}^{\mathrm{VRS}}$ and $\epsilon_{o}^{\mathrm{CRS}}$ the effective bounds for DMU $\left(X_{o}, Y_{o}\right)$ in the single-stage output-oriented VRS and CRS programs, respectively. Similarly, denote $\tilde{\epsilon}_{o}^{\mathrm{VRS}}$ and $\tilde{\epsilon}_{o}^{\mathrm{CRS}}$ the effective bounds in the respective input-oriented VRS and CRS models.

Proposition 7. $\epsilon_{o}^{\mathrm{CRS}} \leq \epsilon_{o}^{\mathrm{VRS}}, \tilde{\epsilon}_{o}^{\mathrm{CRS}} \leq \tilde{\epsilon}_{o}^{\mathrm{VRS}}$.

\section{Examples}

Below we consider two examples that illustrate theoretical results obtained in this paper.

Example 1. Consider the CRS technology with a single input and two outputs generated by observed DMUs $A, B$ and $C$ shown in Table 1 . The other DMUs in this table are also in this technology and may be viewed as observed or unobserved. The last two columns of Table 1 show the value $\varphi_{o}$ evaluated by solving program (7) and the corresponding effective bound $\epsilon_{o}=1 / \varphi_{o}$ (rounded to 5 decimal places).

Figure 1 provides a graphical illustration of this example. The shaded area represents the section of the above technology for the input equal to 1. Each DMU in this graph is shown together with the corresponding value $\varphi_{o}$ (the value in parentheses).

Note that the output radial efficiency of DMU $D$ is equal to 0.5 , and its radial target is DMU $G$. Similarly, the output radial efficiency of DMU $E$ is equal to 0.6 , and its radial target is DMU $B$. The effective bounds $\epsilon_{o}$ for these DMUs shown in Table 1 clearly comply with the statement of Proposition 5. 
Table 1: DMUs in Example 1 and values $\varphi_{o}$ and $\epsilon_{o}$.

\begin{tabular}{llllll}
\hline DMU & Input & Output 1 & Output 2 & $\varphi_{o}$ & $\epsilon_{o}=1 / \varphi_{o}$ \\
\hline$A$ & 1 & 3 & 6 & 15 & 0.06667 \\
$B$ & 1 & 5 & 5 & 10 & 0.1 \\
$C$ & 1 & 6 & 1 & 25 & 0.04 \\
$D$ & 1 & 1 & 3 & 7 & 0.14286 \\
$E$ & 1 & 3 & 3 & 6 & 0.16667 \\
$F$ & 1 & 1 & 6 & 13 & 0.07692 \\
$G$ & 1 & 2 & 6 & 14 & 0.07143 \\
$H$ & 1 & 4 & 5.5 & 15 & 0.06667 \\
$K$ & 1 & 5.5 & 3 & 25 & 0.04 \\
\hline
\end{tabular}

To illustrate the theory developed in this paper, we solve the single-stage program (3) for each DMU, using three different values of $\epsilon$, namely, $0.1,0.05$ and 0.03 . Table 2 shows the corresponding optimum values $\eta^{\epsilon}$ (rounded to 5 decimal places).

Note that $\epsilon=0.03$ is strictly smaller than the effective bound $\epsilon_{o}$ of each DMU (shown in the last column of Table 1). Therefore, according to Theorem 1, the corresponding value $\eta^{\epsilon}$ is the true value of output expansion factor $\eta^{*}$ of each DMU. ${ }^{9}$ The optimal input and output slacks $S_{X}^{\epsilon}$ and $S_{Y}^{\epsilon}$ also coincide with their true values $S_{X}^{*}$ and $S_{Y}^{*}$ obtained by solving program (2) (these are not shown but can be verified by observation of Figure 1).

In line with Theorem 1 , for $\epsilon=0.05$ and $\epsilon=0.1$, we have $\eta^{\epsilon} \neq \eta^{*}$, in all cases where $\epsilon$ is larger than the required effective bound $\epsilon_{o}$. For example, for DMU $A, \epsilon_{o}=0.06667$. This means that computations with $\epsilon=0.05$ produce the true output expansion factor $\eta^{\epsilon}=\eta^{*}=1$, while computations with $\epsilon=0.1$ produce $\eta^{\epsilon}=0.83333<1=\eta^{*}$. (Note that this is consistent with Proposition 3.)

According to Proposition 4, solving the single-stage program (3) with $\epsilon=\epsilon_{o}$ does not guarantee that $\eta^{\epsilon}=\eta^{*}$. This result is relevant for DMU $B$ when we use $\epsilon=0.1$, which coincides with $\epsilon_{o}$ for this DMU. In our computations we obtained $\eta^{\epsilon}=\eta^{*}=1$. In order to illustrate Proposition 4 , we incorporate the additional constraint $\eta+\epsilon\left(\mathbf{1}^{\top} S_{X}+\mathbf{1}^{\top} S_{Y}\right)=1$ in program (3) and change its objective to the minimization of $\eta$. Computations show that the resulting program has an unbounded optimum value. This implies that the original single-stage program (3) has multiple optimal solutions (5), in which $\eta^{\epsilon}$ can be any value from the interval $(-\infty, 1]$.

Similarly, performing additional computations with $\epsilon=0.04$ (not shown) produces the same results as with $\epsilon=0.03$, for all DMUs. However, because $\epsilon_{o}=0.04$ for DMUs $C$ and $K$, by Proposition 4 , there exist alternative optimal solutions to the corresponding singlestage programs (3) in which $\eta^{\epsilon}<\eta^{*}=1$. Computations show that, for DMU $C$, there exist optimal solutions (5) in which $\eta^{\epsilon}$ is any value from the interval $[0.83333,1]$. For DMU $K$, any value $\eta^{\epsilon} \in[0.90909,1]$ can be obtained.

Example 2. It may appear that we can evaluate the effective bounds $\epsilon_{o}$ for all strongly efficient observed DMUs, define $\bar{\epsilon}_{o}$ as the smallest among them, and use $\bar{\epsilon}_{o}$ as a universal

\footnotetext{
${ }^{9}$ The true values $\eta^{*}$ are also easy to compute by observation of Figure 1.
} 


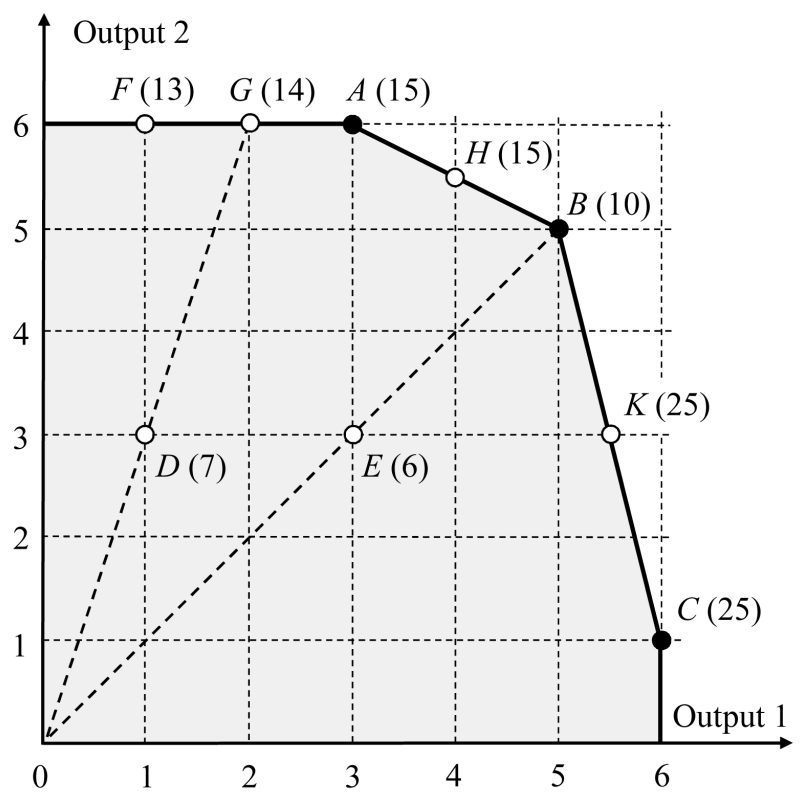

Figure 1: An illustration of Example 1. The values in parentheses show $\varphi_{o}=1 / \epsilon_{o}$ evaluated at the corresponding DMU.

effective bound for all DMUs in the technology. In particular, Figure 1 appears to suggest that such an approach might be valid. ${ }^{10}$ The following example shows that, in the general case, this approach would be incorrect. In particular, single-stage computations for an inefficient DMU may require a smaller $\epsilon$ than for any of the efficient DMUs.

Consider the CRS or VRS technology generated by three observed DMUs $A, B$ and $C$ shown in Table 3, all of which are strongly efficient in both technologies. DMU $D$ is the convex combination of $A, B$ and $C$ with the weights $0.5,0.25$ and 0.25 , respectively, and is also strongly efficient. DMU $E$ is inefficient. Its output radial efficiency is equal to 0.9 in both CRS and VRS technologies, and its efficient radial target is DMU $D$.

The last two columns of Table 3 show effective bounds $\epsilon_{o}^{\mathrm{CRS}}$ and $\epsilon_{o}^{\mathrm{VRS}}$ for the single-stage output-oriented CRS and VRS models. The former are calculated by solving program (7), and the latter by solving program (15).

Note that the effective bounds $\epsilon_{o}$ for the inefficient DMU $E$ and its efficient output radial projection $D$ are strictly smaller than for any of the three efficient observed DMUs $A, B$ and $C$. This observation is true in both the CRS and VRS technologies.

Furthermore, as an illustration to Proposition 7, note that the effective bounds $\epsilon_{o}$ calculated under the assumption of VRS do not exceed those calculated for the CRS technology.

\section{Conclusion}

The single-stage approach to assessing the efficiency of DMUs is usually viewed as an approximate method that, additionally, may suffer from significant computational errors. In this paper we prove that, if the value $\epsilon$ employed by the method is sufficiently small, the single-stage approach theoretically (i.e., assuming we can solve the program exactly, without any computational errors) produces the true radial efficiency of $\mathrm{DMU}_{o}$ and the corresponding

\footnotetext{
${ }^{10}$ In this case, the lowest effective bound $\epsilon_{o}=1 / \varphi_{o}$ is attained at the observed DMU $C$. Therefore, $\bar{\epsilon}_{o}=0.04$. As discussed, in this example, any smaller value, e.g., $\epsilon=0.03$, is suitable for single-stage solutions for all DMUs.
} 
Table 2: Optimal $\eta^{\epsilon}$ for different values $\epsilon$ in Example 1.

\begin{tabular}{llll}
\hline DMU & $\epsilon=0.1$ & $\epsilon=0.05$ & $\epsilon=0.03$ \\
\hline$A$ & 0.83333 & 1 & 1 \\
$B$ & 1 & 1 & 1 \\
$C$ & 0.83333 & 0.83333 & 1 \\
$D$ & 2 & 2 & 2 \\
$E$ & 1.66667 & 1.66667 & 1.66667 \\
$F$ & 0.83333 & 1 & 1 \\
$G$ & 0.83333 & 1 & 1 \\
$H$ & 0.90909 & 1 & 1 \\
$K$ & 0.90909 & 0.90909 & 1 \\
\hline
\end{tabular}

Table 3: DMUs in Example 2 and values $\epsilon_{o}$ in the CRS and VRS technologies.

\begin{tabular}{lllllll}
\hline DMU & Input & Output 1 & Output 2 & Output 3 & $\epsilon_{o}^{\mathrm{CRS}}$ & $\epsilon_{o}^{\text {VRS }}$ \\
\hline$A$ & 1 & 4 & 1 & 1 & 0.02381 & 0.06818 \\
$B$ & 1 & 1 & 4 & 1 & 0.02381 & 0.06818 \\
$C$ & 0.5 & 1 & 1 & 10 & 0.08333 & 0.08333 \\
$D$ & 0.875 & 2.5 & 1.75 & 3.25 & 0.01191 & 0.05985 \\
$E$ & 0.875 & 2.25 & 1.575 & 2.925 & 0.01323 & 0.06650 \\
\hline
\end{tabular}

optimal slacks, exactly the same as obtained by the two-stage approach of Ali and Seiford (1993a).

We prove that the threshold value $\epsilon_{o}$, referred to as the effective bound, can be computed by solving a specially constructed linear program for each $\mathrm{DMU}_{o}$. Such a linear program requires that we know the true input or output radial efficiency of $\mathrm{DMU}_{o}$ and the corresponding optimal input and output slacks. This means that we implement the two-stage procedure before we identify the effective bound $\epsilon_{o}$, which leaves the subsequent single-stage implementation uninteresting from the practical perspective.

However, our results provide a useful insight into the asymptotic behaviour of efficiency assessed by the single-stage method as a function of $\epsilon$. In particular, they allow us to explain the behaviour of the output improvement factor $\eta^{\epsilon}$ as a function of $\epsilon$ shown in Table 2 of the illustrative example.

Out results imply that, if we reduce $\epsilon$ aiming to improve the precision of the single-stage solution (viewed as an approximation of the true solution yielded by the two-stage method), then there exists the threshold value $\epsilon_{o}>0$ below which the approximation error is not improved, because the theoretically precise (true) solution is already achieved. Therefore, for very small values $\epsilon$, any discrepancy between the calculated and true efficiencies of $\mathrm{DMU}_{o}$ is explained entirely by computational errors.

Expressing the effective bound $\epsilon_{o}$ as the inverse optimal value of a specially constructed 
linear program leads to further theoretical results. In particular, we prove that the effective bound for any $\mathrm{DMU}_{o}$ in the CRS model does not exceed its effective bound in the VRS model, in both input and output orientations. We also show that the effective bounds in the input and output orientations, for both CRS and VRS models, are generally different, and their evaluation requires solving different linear programs. We show by an example that the effective bound of an inefficient DMU may be smaller than the efficient bound of any of the strongly efficient DMUs. This implies that assessing the effective bounds for all observed DMUs is generally insufficient to identify the common effective value $\epsilon$ that would be suitable for all (including unobserved) DMUs in the CRS or VRS technology.

Another implication of our results concerns interpretation of single-stage DEA models. Traditionally, the value $\epsilon$ in these models is regarded as a non-Archimedean infinitesimal whose definition is, as highlighted by Cooper et al. (2007, page 74), based on non-standard mathematics of linear programming. Our results show that we can always stay within the realm of standard linear programming and interpret $\epsilon$ as a sufficiently small positive real number.

From a practical perspective, considering the importance of the effective bound $\epsilon_{o}$ for single-stage models, it would be interesting to develop a method for assessing or approximating $\epsilon_{o}$ that does not require an explicit knowledge of the radial efficiency of $\mathrm{DMU}_{o}$ and the corresponding maximum slacks. Addressing this issue is a topic open for future research. 


\section{Appendix A. Proofs}

Proof of Proposition 1. By Definition 1, $\eta^{\epsilon}=\eta^{*}$. Therefore, $\left\langle\lambda^{\epsilon}, S_{X}^{\epsilon}, S_{Y}^{\epsilon}\right\rangle$ is an optimal solution to program (3) with the additional constraint $\eta=\eta^{*}$. This makes $\eta$ a fixed constant, and maximizing the objective function (3a) is equivalent to maximizing the objective function of program (2).

Conversely, let $\left\langle\lambda^{*}, S_{X}^{*}, S_{Y}^{*}\right\rangle$ be an optimal solution to $(2)$. Then $\left\langle\eta^{*}, \lambda^{*}, S_{X}^{*}, S_{Y}^{*}\right\rangle$ is a feasible solution to program (3). Consider any optimal solution (5) to program (3). By Definition $1, \eta^{\epsilon}=\eta^{*}$. Then $\left\langle\lambda^{\epsilon}, S_{X}^{\epsilon}, S_{Y}^{\epsilon}\right\rangle$ is an optimal solution to program (2). Therefore, $\mathbf{1}^{\top} S_{X}^{\epsilon}+\mathbf{1}^{\top} S_{Y}^{\epsilon}=\mathbf{1}^{\top} S_{X}^{*}+\mathbf{1}^{\top} S_{Y}^{*}$, and $\left\langle\eta^{*}, \lambda^{*}, S_{X}^{*}, S_{Y}^{*}\right\rangle$ is an optimal solution to (3).

Proof of Proposition 2. The fact that program (7) has a finite optimum value is established in the proof of Theorem 1 . The inequality $\varphi_{o} \geq Y_{o}^{\top} \mathbf{1}$ follows from $(7 \mathrm{~d})$.

Proof of Theorem 1. Let $\eta^{*}$ be the optimum value of program (1), and let $\left\langle\lambda^{*}, S_{X}^{*}, S_{Y}^{*}\right\rangle$ be an optimal solution to program (2). Then $\left\langle\eta^{*}, \lambda^{*}, S_{X}^{*}, S_{Y}^{*}\right\rangle$ is a feasible solution to program (3), whose objective function is equal to

$$
\eta^{*}+\epsilon\left(\mathbf{1}^{\top} S_{X}^{*}+\mathbf{1}^{\top} S_{Y}^{*}\right)
$$

Consider the following linear program in which $\eta^{*}$ is fixed and $\alpha \geq 0$ is a parameter (not used for optimization):

$$
\begin{aligned}
\Phi(\alpha)=\max & \epsilon\left(\mathbf{1}^{\top} S_{X}+\mathbf{1}^{\top} S_{Y}\right) \\
\text { subject to } & \bar{X} \lambda+S_{X}=X_{o}, \\
& -\bar{Y} \lambda+S_{Y}=-\eta^{*} Y_{o}+\alpha Y_{o}, \\
& \lambda, S_{X}, S_{Y} \geq \mathbf{0} .
\end{aligned}
$$

If $\alpha=0$, program (A.2) is program (2) (with its objective function multiplied by $\epsilon$ ). Therefore,

$$
\Phi(0)=\epsilon\left(\mathbf{1}^{\top} S_{X}^{*}+\mathbf{1}^{\top} S_{Y}^{*}\right)
$$

If $\alpha \neq 0$, program (A.2) can be regarded as a perturbed program (2) (with its objective function multiplied by $\epsilon$ ) in which the vector of perturbation $Y_{o}$ on the right-hand side of constraint (A.2c) is used in proportion $\alpha$.

By Lemma 1, program (A.2) is feasible and has a finite optimum value for all $\alpha \in \Gamma=$ $[0,+\infty)$, i.e., $\Gamma$ is the domain of function $\Phi(\alpha)$. As known from sensitivity analysis in linear optimization (see, e.g, Roos et al. 2005, Theorems IV.48 and IV.50), $\Phi(\alpha)$ is a continuous, concave and piecewise linear function on $\Gamma$. Because function $\Phi(\alpha)$ is linear in some right neighbourhood of $\alpha=0$, it has a finite right-hand derivative $\Phi_{+}^{\prime}(0)$.

By Lemma 2, program (7) is feasible and its optimum value $\varphi_{o}$ is finite, which proves Proposition 2. Furthermore,

$$
\Phi_{+}^{\prime}(0)=\epsilon \varphi_{o} .
$$

Because $\Phi(\alpha)$ is concave on $\Gamma$, we have

$$
\Phi(\alpha) \leq \Phi(0)+\Phi_{+}^{\prime}(0) \alpha, \quad \forall \alpha \in \Gamma .
$$

Moreover, because function $\Phi(\alpha)$ is linear in some right neighbourhood $[0, \bar{\alpha}]$ of $\alpha=0$ (where $\bar{\alpha}>0$ ), the inequality (A.5) is satisfied as equality for all $\alpha \in[0, \bar{\alpha}]$, i.e.,

$$
\Phi(\alpha)=\Phi(0)+\Phi_{+}^{\prime}(0) \alpha, \quad \forall \alpha \in[0, \bar{\alpha}] .
$$

The proof now follows from Lemma 3 and Propositions 3 and 4. 
Lemma 1. The domain $\Gamma$ of function $\Phi(\alpha)$ is $[0,+\infty)$.

Proof of Lemma 1. Obviously, $0 \in \Gamma$. Let $\left\langle\lambda^{\prime}, S_{X}^{\prime}, S_{Y}^{\prime}\right\rangle$ denote an optimal solution to (A.2), where $\alpha=0$. Let $\tilde{\alpha}>0$. Then $\left\langle\lambda^{\prime}, S_{X}^{\prime}, S_{Y}^{\prime}+\tilde{\alpha} Y_{o}\right\rangle$ is an optimal solution to (A.2), where $\alpha=\tilde{\alpha}$. (In particular, $S_{Y}=S_{Y}^{\prime}+\tilde{\alpha} Y_{o}$ satisfies (A.2c).) Therefore, $\tilde{\alpha} \in \Gamma$. Finally, let $\tilde{\alpha}<0$. We need to prove that program (A.2) is infeasible. Indeed, assume the opposite. Then program (A.2) is feasible for $\tilde{\eta}=\eta^{*}-\tilde{\alpha}>\eta^{*}$.

By constraints (A.2b) and (A.2c), the objective function of program (A.2) is bounded above. Indeed, (A.2b) implies that the terms $\bar{X} \lambda$ and $S_{X}$ are bounded above by vector $X_{o}$. Because all columns $X_{j}$ of matrix $\bar{X}, j=1, \ldots, n$, are nonzero, all components of vector $\lambda$ are bounded above. Then the term $\bar{Y} \lambda$ in (A.2c), and hence $S_{Y}$, are bounded above. Therefore, program (A.2) has a finite optimal solution $\left\langle\tilde{\lambda}, \tilde{S}_{X}, \tilde{S}_{Y}\right\rangle$. The same solution must be optimal in program (2) in which $\eta^{*}$ is replaced by $\tilde{\eta}$. By Remark $1,\left\langle\tilde{\eta}, \tilde{\lambda}, \tilde{S}_{X}, \tilde{S}_{Y}\right\rangle$ is optimal in (1), which contradicts the assumption that $\eta^{*}$ is the optimum value of program (1).

Lemma 2. Program (7) is feasible and has a finite optimum value $\varphi_{o}$. Furthermore, $\Phi_{+}^{\prime}(0)=$ $\epsilon \varphi_{0}$.

Proof of Lemma 2. As shown in the proof of Theorem 1, the right-hand derivative $\Phi_{+}^{\prime}(0)$ exists and is finite. Using the known result of sensitivity analysis, to calculate $\Phi_{+}^{\prime}(0)$, consider the dual to (A.2) where $\alpha=0$ :

$$
\begin{aligned}
\min & X_{o}^{\top} v-\eta^{*} Y_{o}^{\top} u \\
\text { subject to } & \bar{X}^{\top} v-\bar{Y}^{\top} u \geq \mathbf{0}, \\
& u, v \geq \epsilon \mathbf{1}
\end{aligned}
$$

Denote $\Omega$ the set of optimal solutions to (A.7). Then (see, e.g., Roos et al. 2005, Theorem IV.62)

$$
\Phi_{+}^{\prime}(0)=\min \left\{Y_{o}^{\top} u \mid(u, v) \in \Omega\right\} .
$$

Because $\Phi_{+}^{\prime}(0)$ exists and is finite, program (A.8) is feasible and has a finite optimal solution. To restate program (A.8) in an operational form, note that program (A.7) is the dual to (A.2) with $\alpha=0$, and the latter is program (2), with its objective function multiplied by $\epsilon$. Therefore, the optimum value of (A.7) is equal to $\epsilon \sigma^{*}$. Hence, the set of optimal solutions $\Omega$ of program (A.7) is the set of all its feasible solutions for which the objective function of (A.7) is equal to $\epsilon \sigma^{*}$. Then program (A.8) can be stated as follows:

$$
\begin{aligned}
\Phi_{+}^{\prime}(0)=\min & Y_{o}^{\top} u \\
\text { subject to } & X_{o}^{\top} v-\eta^{*} Y_{o}^{\top} u=\epsilon \sigma^{*}, \\
& \bar{X}^{\top} v-\bar{Y}^{\top} u \geq \mathbf{0}, \\
& u, v \geq \epsilon \mathbf{1} .
\end{aligned}
$$

Substituting $\tilde{u}=u / \epsilon$ and $\tilde{v}=v / \epsilon$, we have

$$
\begin{aligned}
\Phi_{+}^{\prime}(0)=\epsilon \times \min & Y_{o}^{\top} \tilde{u} \\
\text { subject to } & X_{o}^{\top} \tilde{v}-\eta^{*} Y_{o}^{\top} \tilde{u}=\sigma^{*}, \\
& \bar{X}^{\top} \tilde{v}-\bar{Y}^{\top} \tilde{u} \geq \mathbf{0} \\
& \tilde{u}, \tilde{v} \geq \mathbf{1}
\end{aligned}
$$

Comparing with (7), we have $\Phi_{+}^{\prime}(0)=\epsilon \varphi_{0}$. Because, as shown, $\Phi_{+}^{\prime}(0)$ is finite, $\varphi_{o}$ is also finite, which completes the proof. 
Lemma 3. Any $\epsilon \in\left(0,1 / \varphi_{o}\right)$ is an effective value for $D M U\left(X_{o}, Y_{o}\right)$.

Proof of Lemma 3. Consider any $\epsilon \in\left(0,1 / \varphi_{o}\right)$. Then, by (A.4),

$$
\Phi_{+}^{\prime}(0)<1
$$

Let $\left\langle\eta^{\epsilon}, \lambda^{\epsilon}, S_{X}^{\epsilon}, S_{Y}^{\epsilon}\right\rangle$ be an optimal solution to (3). We need to prove that $\eta^{\epsilon}=\eta^{*}$. First note that $\eta^{\epsilon} \leq \eta^{*}$. (This follows from the fact that $\left\langle\eta^{\epsilon}, \lambda^{\epsilon}, S_{X}^{\epsilon}, S_{Y}^{\epsilon}\right\rangle$ is feasible in (1) and that $\eta^{*}$ is the optimum value of (1).) Assume that $\eta^{\epsilon}<\eta^{*}$, and let $\hat{\alpha}=\eta^{*}-\eta^{\epsilon}>0$. Then the optimum value of program (3) is

$$
\eta^{\epsilon}+\epsilon\left(\mathbf{1}^{\top} S_{X}^{\epsilon}+\mathbf{1}^{\top} S_{Y}^{\epsilon}\right)=\eta^{\epsilon}+\Phi(\hat{\alpha}) .
$$

Taking into account (A.1) and (A.3), observe that the objective function of program (3) at its feasible solution $\left\langle\eta^{*}, \lambda^{*}, S_{X}^{*}, S_{Y}^{*}\right\rangle$ is equal to

$$
\eta^{*}+\epsilon\left(\mathbf{1}^{\top} S_{X}^{*}+\mathbf{1}^{\top} S_{Y}^{*}\right)=\eta^{*}+\Phi(0) .
$$

Subtracting the right-hand side of (A.11) from the right-hand side of (A.10), and noting (A.5) and (A.9), we obtain

$$
\eta^{\epsilon}-\eta^{*}+\Phi(\hat{\alpha})-\Phi(0) \leq-\hat{\alpha}+\Phi_{+}^{\prime}(0) \hat{\alpha}=\left(\Phi_{+}^{\prime}(0)-1\right) \hat{\alpha}<0 .
$$

This contradicts the assumption that $\left\langle\eta^{\epsilon}, \lambda^{\epsilon}, S_{X}^{\epsilon}, S_{Y}^{\epsilon}\right\rangle$ is an optimal solution to (3). Therefore, $\eta^{\epsilon}=\eta^{*}$.

Proof of Proposition 3. Let $\epsilon \in\left(1 / \varphi_{o}, \epsilon^{*}\right)$ and, therefore, program (3) has a final optimum value. To prove Proposition 3, it suffices to prove the following two statements.

(i) There exists a feasible solution $\left\langle\tilde{\eta}, \tilde{\lambda}, \tilde{S}_{X}, \tilde{S}_{Y}\right\rangle$ to program (3) for which its objective function is strictly larger than for $\left\langle\eta^{*}, \lambda^{*}, S_{X}^{*}, S_{Y}^{*}\right\rangle$, i.e.,

$$
\tilde{\eta}+\epsilon\left(\mathbf{1}^{\top} \tilde{S}_{X}+\mathbf{1}^{\top} \tilde{S}_{Y}\right)>\eta^{*}+\epsilon\left(\mathbf{1}^{\top} S_{X}^{*}+\mathbf{1}^{\top} S_{Y}^{*}\right)
$$

Therefore, the inequality (A.12) is true for any optimal solution $\left\langle\tilde{\eta}, \tilde{\lambda}, \tilde{S}_{X}, \tilde{S}_{Y}\right\rangle$ to program (3).

(ii) For any optimal solution $\left\langle\tilde{\eta}, \tilde{\lambda}, \tilde{S}_{X}, \tilde{S}_{Y}\right\rangle$ to program (3), we have $\tilde{\eta}<\eta^{*}$.

To prove statement (i), first note that, by (A.4),

$$
\Phi_{+}^{\prime}(0)>1 \text {. }
$$

Consider any $\tilde{\eta}<\eta^{*}$ such that $\tilde{\alpha}=\eta^{*}-\tilde{\eta} \in[0, \bar{\alpha}]$, where $\bar{\alpha}>0$ is as defined in the proof of Theorem 1. Let $\left\langle\tilde{\lambda}, \tilde{S}_{X}, \tilde{S}_{Y}\right\rangle$ be an optimal solution to program (A.2), where $\alpha=\tilde{\alpha}$. Then $\left\langle\tilde{\eta}, \tilde{\lambda}, \tilde{S}_{X}, \tilde{S}_{Y}\right\rangle$ is a feasible solution to program (3), for which its objective function is equal to

$$
\tilde{\eta}+\epsilon\left(\mathbf{1}^{\top} \tilde{S}_{X}+\mathbf{1}^{\top} \tilde{S}_{Y}\right)=\tilde{\eta}+\Phi(\tilde{\alpha})
$$

Because $\Phi(\alpha)$ is a linear function on $[0, \bar{\alpha}],($ A.6) implies

$$
\Phi(\tilde{\alpha})=\Phi(0)+\Phi_{+}^{\prime}(0) \tilde{\alpha} .
$$

The value of the objective function of (3) at $\left\langle\eta^{*}, \lambda^{*}, S_{X}^{*}, S_{Y}^{*}\right\rangle$ is given by (A.11). Subtracting the right-hand side of (A.11) from the right-hand side of (A.14), and using the substitution of (A.15), we have

$$
[\tilde{\eta}+\Phi(\tilde{\alpha})]-\left[\eta^{*}+\Phi(0)\right]=-\tilde{\alpha}+\Phi_{+}^{\prime}(0) \tilde{\alpha}=\left(\Phi_{+}^{\prime}(0)-1\right) \tilde{\alpha} .
$$


Statement (i) now follows from (A.13).

To prove statement (ii), consider any optimal solution $\left\langle\tilde{\eta}, \tilde{\lambda}, \tilde{S}_{X}, \tilde{S}_{Y}\right\rangle$ to program (3). By statement (i), this solution satisfies (A.12). Taking into account (6) (where $\eta^{\epsilon}$ is replaced by $\tilde{\eta}$ ), it suffices to prove that the case $\tilde{\eta}=\eta^{*}$ is impossible, and therefore we have $\tilde{\eta}<\eta^{*}$.

Indeed, assume that $\tilde{\eta}=\eta^{*}$. Then $\left\langle\tilde{\lambda}, \tilde{S}_{X}, \tilde{S}_{Y}\right\rangle$ is feasible in program (2). From (A.12) we have

$$
\mathbf{1}^{\top} \tilde{S}_{X}+\mathbf{1}^{\top} \tilde{S}_{Y}>\mathbf{1}^{\top} S_{X}^{*}+\mathbf{1}^{\top} S_{Y}^{*}
$$

which contradicts the optimality of solution $\left\langle\lambda^{*}, S_{X}^{*}, S_{Y}^{*}\right\rangle$ in program (2).

Proof of Proposition 4. First note that, if $\epsilon=1 / \varphi_{o}$, we have

$$
\Phi_{+}^{\prime}(0)=1
$$

As in the proof of Lemma 3, it is straightforward to show that $\left\langle\eta^{*}, \lambda^{*}, S_{X}^{*}, S_{Y}^{*}\right\rangle$ is an optimal solution to program (3).

Let $\bar{\alpha}$ be as defined in the proof of Theorem 1 . For any $\tilde{\alpha} \in(0, \bar{\alpha}]$, consider the feasible solution $\left\langle\tilde{\eta}, \tilde{\lambda}, \tilde{S}_{X}, \tilde{S}_{Y}\right\rangle$ to program (3), defined in the proof of Proposition 3. For this solution, because $\eta^{*}-\tilde{\eta}=\tilde{\alpha}>0$, we have $\tilde{\eta}<\eta^{*}$. Subtracting the value of the objective function of program (3) at solution $\left\langle\eta^{*}, \lambda^{*}, S_{X}^{*}, S_{Y}^{*}\right\rangle$ from its value at solution $\left\langle\tilde{\eta}, \tilde{\lambda}, \tilde{S}_{X}, \tilde{S}_{Y}\right\rangle$ (i.e., subtracting (A.11) from (A.14)), we obtain (A.16). Using the substitution of (A.17), we have

$$
[\tilde{\eta}+\Phi(\tilde{\alpha})]-\left[\eta^{*}+\Phi(0)\right]=\left(\Phi_{+}^{\prime}(0)-1\right) \tilde{\alpha}=0 .
$$

Therefore, $\left\langle\tilde{\eta}, \tilde{\lambda}, \tilde{S}_{X}, \tilde{S}_{Y}\right\rangle$ is an optimal solution to program (3), and $\tilde{\eta}<\eta^{*}$.

Proof of Proposition 5. For DMU $(\hat{X}, \hat{Y})$, the optimum value $\hat{\eta}^{*}$ of the corresponding first-stage program (1) is equal to 1 , and the maximum sum of slacks in the corresponding program (2) is equal to $\sigma^{*}$, the same as for DMU $\left(X_{o}, Y_{o}\right)$. Therefore, the single-stage program for $\operatorname{DMU}(\hat{X}, \hat{Y})$ is program $(7)$ in which the objective function is changed to $\eta^{*} Y_{o}^{\top} u$. For its optimum value $\hat{\varphi}_{o}$ we have $\hat{\varphi}_{o}=\eta^{*} \varphi_{o}$. This implies $\hat{\epsilon}_{o}=\epsilon_{o} / \eta^{*}$.

Proof of Proposition 6. Because DMU $\left(X_{o}, Y_{o}\right)$ is strongly efficient, in program (13), $\theta^{*}=1$ and $\sigma^{*}=0$. Then program (13) is program (8), and $\varphi_{o}=\tilde{\varphi}_{o}$.

Proof of Proposition 7. Let $\left\langle u^{\prime}, v^{\prime}\right\rangle$ be an optimal solution to program (7). Then $\left\langle u^{\prime}, v^{\prime}, \omega^{\prime}\right\rangle$, where $\omega^{\prime}=0$, is feasible in program (15). The objective functions of these two programs are equal for the two solutions. Therefore, $\varphi_{o}^{\mathrm{CRS}} \geq \varphi_{o}^{\mathrm{VRS}}$, where $\varphi_{o}^{\mathrm{CRS}}$ is the optimum value of (7) and $\varphi_{o}^{\mathrm{VRS}}$ is the optimum value of (15). Similarly, $\tilde{\varphi}_{o}^{\mathrm{CRS}} \geq \tilde{\varphi}_{o}^{\mathrm{VRS}}$. 


\section{References}

Ali, A.I., Seiford, L.M. (1993a). The mathematical programming approach to efficiency analysis. In H.O. Fried, C.A.K. Lovell, S.S. Schmidt (Eds.), The measurement of productive efficiency: Techniques and applications (pp. 120-159). New York: Oxford University Press.

Ali, A.I., Seiford, L.M. (1993b). Computational accuracy and infinitesimals in data envelopment analysis. Information Systems and Operational Research, 31, 290-297.

Alirezaee, M.R. (2005). The overall assurance interval for the non-Archimedean epsilon in DEA models: a partition base algorithm. Applied Mathematics and Computation, 164(3), 667-674.

Amin, G.R., Toloo, M. (2004). A polynomial-time algorithm for finding $\varepsilon$ in DEA models. Computers \& Operations Research, 31(5), 803-805.

Banker, R.D., Charnes, A., Cooper, W.W. (1984). Some models for estimating technical and scale efficiencies in data envelopment analysis. Management Science, 30(9), 1078-1092.

Boyd, G., Färe, R. (1984). Measuring the efficiency of decision making units: A comment. European Journal of Operational Research, 15(3), 331-332.

Charnes, A., Cooper, W.W. (1984). The non-archimedean CCR ratio for efficiency analysis: A rejoinder to Boyd and Färe. European Journal of Operational Research, 15(3), 333-334.

Charnes, A., Cooper, W.W., Rhodes, E. (1978). Measuring the efficiency of decision making units. European Journal of Operational Research, 2(6), 429-444.

Charnes, A., Cooper, W.W., Rhodes, E. (1979). Measuring the efficiency of decision making units. European Journal of Operational Research, 3(4), 339.

Charnes, A., Rousseau, J.J., Semple, J. (1992). Non-archimedean infinitesimals, transcendentals and categorical inputs in linear programming and data envelopment analysis. International Journal of Systems Science, 23(12), 2401-2406.

Charnes, A., Rousseau, J., Semple, J. (1993). An effective non-Archimedean anti-degeneracy/cycling linear programming method especially for data envelopment analysis and like models. Annals of Operations Research, 46-47(2), 271-278.

Cooper, W.W., Seiford, L.M., Tone, K. (2007). Data envelopment analysis. A comprehensive text with models, applications, references and DEA-Solver software (2nd ed.). New York: Springer Science + Business Media.

Mehrabian, S., Jahanshahloo, G.R., Alirezaee, M.R., Amin, G.R. (2000). An assurance interval of the nonArchimedean epsilon in DEA models. Operations Research, 48(2), 344-347.

MirHassani, S.A., Alirezaee, M.R. (2005). An efficient approach for computing non-Archimedean $\varepsilon$ in DEA based on integrated models. Applied Mathematics and Computation, 166(2), 449-456.

Podinovski, V.V. (2004). Production trade-offs and weight restrictions in data envelopment analysis. Journal of the Operational Research Society, 55(12), 1311-1322.

Podinovski, V.V. (2005). The explicit role of weight bounds in models of data envelopment analysis. Journal of the Operational Research Society, 56(12), 1408-1418.

Podinovski, V.V. (2016). Optimal weights in DEA models with weight restrictions. European Journal of Operational Research, 254(3), 916-924.

Podinovski, V.V., Bouzdine-Chameeva, T. (2013). Weight restrictions and free production in data envelopment analysis. Operations Research, 61(2), 426-437.

Podinovski, V.V., Bouzdine-Chameeva, T. (2015). Consistent weight restrictions in data envelopment analysis. European Journal of Operational Research, 244(1), 201-209.

Roos, C., Terlaky, T., Vial, J.P. (2005). Interior point methods for linear optimization. New York: Springer Science + Busines Media.

Scheel, H., Scholtes, S. (2003). Continuity of DEA efficiency measures. Operations Research, 51(1), 149-159. 